\title{
RECENT ACHIEVEMENTS FOR IMPROVING REPRODUCTIVE EFFICIENCY OF DROMEDARY CAMELS IN EGYPT
}

\author{
Sh.A. Rateb *, Kh. A. El-Bahrawy, Marwa A. Khalifa, I.S. Abd El-Hamid, A.A. Zaghloul, A.M. \\ Kamel and E.E. El-Hassanein
}

Animal and Poultry Production Division, Desert Research Center, Ministry of Agriculture and Land Reclamation, Egypt, * Corresponding author: E-mail: rateb.drc@gmail.com

Received: 4/9/2019 Accepted: 20/10/2019

\section{SUMMARY}

Over the past decade, the realization of camel importance increased exponentially, not only in the Arabian world, but also by developmental organizations and decision makers all over the globe. Versatility of camels to survive and produce under escalating desertification and drastic environmental conditions has drawn the attention to this uniquely-adapted animal. Consequently, research on camels was motivated in different areas to compensate a long period of neglecting this specie compared to other domestic animals. The low reproductive efficiency of camels, particularly those reared under poor pastoral systems, has been well emphasized. In recent years, our research team at Laboratory of Artificial Insemination and Embryo Transfer, Mariout Research Station, Alexandria, Egypt strived to enhance reproductive performance of camels by developing assisted reproductive techniques. For instance, improving cryopreservation capacity of spermatozoa via enriching the diluents with additives; as well by developing procedures to eliminate semen viscosity has been addressed. With respect to females, new techniques and modalities for induction of synchronized multiple ovulation were expanded, while practical in-field estrus detection was, for the first time, appropriately determined. Furthermore, employing sophisticated molecular genetics techniques to identify potential fertility of males was endeavored. Developing in vitro fertilization procedure and non-conventional embryo production by intracytoplasmic sperm injection (ICSI)were also disclosed. In the present review we aimed to spot the light on recent achievements for enhancing camel reproductive efficiency in Egypt. Potential future research prospects were also proposed.

Keywords: Camels, estrus detection, superovulation, cryopreservation, viscosity elimination

\section{INTRODUCTION}

Developing and application of assisted reproductive techniques over years of extensive research contributed substantially in improving modern livestock industry. Although Camelus dromedarius is regarded as the most versatile specie to produce and reproduce under sever environmental conditions, it did not receive the same attention on different aspects of research as with other domestic animals. Until the early years of the past decade, research on dromedaries was limited and focused mainly on its adaptability characteristics as wellon its potential as a racing animal particularly in the Gulf area. As camel importance was later recognized worldwide, research was expanded in different areas including reproduction. In Egypt, for example, several constraints have been associated with limited reproductive efficiency of camels (El-Hassanein, 2003). Consequently, great efforts were exerted over the past years to enhance dromedaries' reproductive capacity(El-Bahrawy et al., 2015). Perpetuating our quest, the present article aims to congregate our most recent contribution in this topic with respect to both male and female camel reproduction.

\section{Enhancing reproductive efficiency of male dromedaries \\ Improving cryopreservation potential of spermatozoa}

Successful application of assisted reproductive techniques (ART's); such as artificial insemination, in vitro fertilization and embryo transfer, depends fundamentally on the biophysical traits of obtained ejaculates and its potential for processing with minimum loss of sperm fertilization capacity. Accordingly, several investigations were implemented to enhance liquid chilled- and cryopreservation of camel spermatozoa. In this context, El-Bahrawy et al. (2006) evaluated different diluents, i.e. tris-sucrose, tris-citrate, lactose, skim-milk and sucrose, for their potential to maintain sperm viability criteria following chilled- and cryo-preservation. They concluded that a glycerolized ( $2 \%$ glycerol, $\mathrm{v} / \mathrm{v})$ tris-lactose medium was most appropriate. Furthermore, El-Bahrawy et al. (2010) reported that incorporating $400 \mu \mathrm{g} / \mathrm{mL}$ ciprofloxacin efficiently subdued microbial contamination of cryopreserved semen without affecting post-thaw sperm properties. Later on, El-Bahrawy et al. (2012) proposed a complete semen collection and processing regimen that can be reliably applied in laboratories dealing with camel semen to produce adequate cryopreserved 
doses. This regimen comprised a 2-times weekly collection sessions schedule followed by diluting the obtained ejaculates with tris-lactose medium and, further, hawing cryopreserved doses rapidly $\left(65^{\circ} \mathrm{C}\right.$ for 10 seconds). Most recently, El-Bahrawy (2017 $a$ ) noted that supplementing the aforementioned diluent with caffeine $(4 \mathrm{mM})$ enhanced post-thaw recovery of cryopreserved camel spermatozoa and reduced the percent of sperm abnormalities while maintaining acrosome integrity.

\section{Elimination of semen viscosity}

Unlike other domestic species, the dromedary camel semen is remarkably viscous. Viscosity of camel seminal plasma has been attributed to presence of mucopolysaccharides and/or proteins which are secreted from the bulbourethral gland or the prostate upon ejaculation (Mosaferi et al., 2005; KershawYoung et al., 2013). Although this semen feature differs in consistency from one male to another, it plays an influential rule in camel natural breeding. However, it is considered one of the major impediments for development of ART's in camels. Therefore, developing efficient methodologies to diminish camel semen viscosity prior to processing became insistent.

Over the last two decades, several techniques were developed to reduce/eliminate viscosity of camelidea semen and, consequently, enhance sperm motility. In Egypt, for instance, El-Bahrawy (2010a) efficiently utilized the mechanical effect of centrifugation to reduce camel semen viscosity. Meanwhile, incorporating various enzymes that act via different pathways into sperm diluent was investigated for their potential in eliminating dromedary semen viscosity. Based on the hypothesis of attributing semen viscosity to presence of mucopolysaccharides in seminal plasma, a long-chain carbohydrates break-down reagent (i.e. $\alpha$-amylase) was found effective in achieving the goal prior to processing (El-Bahrawy, 2010b). On the other hand, adopting the hypothesis of attribution of proteins in the seminal plasma as the primary determinant of viscosity, supplementing sperm medium with different proteases that manifest peptidase-like activity (i.e. papain and bromelain) efficiently reduced semen viscosity and improved motility and kinematics of cryopreserved spermatozoa (ElBahrawy et al., 2017). Moreover, both mechanical and enzymatic techniques were successfully employed in concert to reduce camel semen viscosity (El-Bahrawy, 2017 ). Most recently, however, we demonstrated how these proteases drastically affected sperm vitality, acrosome integrity and sperm cell surface glycocalyx pattern particularly after the freezing/thawing cycle (Rateb et al., 2019). Alternatively, we investigated the effect of exposing diluted-raw camel semen to high-power lowfrequency ultrasound waves on eliminating viscosity. It was concluded that the ultrasonic wavesaptly reduced semen viscosity and enhanced sperm traits with minimal drastic effects on vitality and cell membrane integrity of liquid-chilled- (Rateb, 2016) and cryo-preserved (El-Bahrawy et al., 2017) spermatozoa.

\section{Sophisticated fertility-related practices}

In general, research on dromedary bulls' infertility has been addressed with emphasis on causes, effects and alleviation. Nonetheless, reasons for issues such as delayed puberty in the males and absence of rut in adult bulls required further attention to be determined (Rateb et al., 2011; El-Bahrawy et al., 2012).

Considering the fact that determinants of fertility are assorted into expressed and inherent, with the later referring to the genetic potential for reproductive performance, the fluorescently-labeled differential display PCR technique was recently utilized to visualize expression of potential fertilityrelated molecular markers relative to semen traits and prolificacy in three categories of mature camel bulls; i.e. high, medium and low prolific (Saad et al., 2019). The visualized chromatograms of differentially displayed fragments evinced detection of 24 seasonally-expressed mRNA molecules; of which 5 were expressed off the breeding season (downregulated), and 19 molecules were expressed at the peak of rutting period (up-regulated). Further, the expression of the later fragments varied corresponding to the prolific index of the male, which may allow for determining, on molecular genetics bases, fertility of adult bulls or even further to predict potential fertility of pre-pubertal calves.

\section{Enhancing reproductive efficiency of female dromedaries \\ In-field estrus detection}

Detection of estrus is considered a mainstay in modern livestock industry. Over the years, several methods were developed to accurately detect estrus, particularly in spontaneous ovulators, based on certain physiological, endocrinological and behavioural peculiarities. In induced ovulators such as camels, however, accurate in-field estrus detection by visual observation was considered very difficult, and relied mainly on receptivity of the female to mating. Nevertheless, none of previous studies revealed undisputable estrus attributes in dromedary females to rely on when determining the optimum timing for natural mating/artificial insemination. This is the reason for why utilizing ultrasonography to monitor ovarian follicular activity and determine the phase of the ovarian cycle became a must.

In our most recent work in this topic, Padalino et al. (2016) reported that during the mature phase of the ovarian cycle (ovulatory phase) female dromedaries exhibit clear behavioral patternsin presence of a restrained bull which could be used as estrus indicators. The authors observed that, in presence of mature ovarian follicles, the females tended to solicit the attention of the male and showed more interest in him by: voluntarily standing close to the male for longer periods (male time score), 
seeking out and interacting with the male more, and showing a clear sign of receptivity by willingly taking the recumbence position of natural mating in front of him.

\section{Application of Assisted Reproductive Techniques (ART's)}

Basically, dromedary females are considered seasonally polyestrous with ceased ovarian activity outside the breeding season (December through March). Off season in Egypt, the ovaries become inactive or, at best, bear few small recruiting follicles $(\varnothing \leq 4 \mathrm{~mm})$ that rarely attain maturity $(12 \mathrm{~mm}<$ follicle $\varnothing<19 \mathrm{~mm}$ ) (Padalino et al., 2016). Hence, preparing female dromedaries for application of artificial insemination and embryo transfer outside the breeding season became challenging and gained focus of recent research.

\section{Hormonal induction of synchronized multiple ovulation}

Multiple ovulation is a substantial prerequisite in ET programs, and aims to generate considerable number of follicles in donor females. In dromedaries, various hormonal protocols were emphasized to induce multiple ovulation either during or off the breeding season. Commonly, these protocols implicated administering exogenous follicle stimulating hormone (FSH) of different origins or hormones that exhibit similar effect to stimulate ovarian activity (Anouassi and Tibary, 2013). Regardless of applying such gonadotropin treatment in succession to a period of progesterone priming or not, previous studies reported substantial variations in ovarian response due to the differences in gonadotropin dosage as well as fashion of administration.

In this respect, Deen and El-Hassanein (2013) reported that administration of the gonadotropin in a single shot one day before or on the day of completing a period of progesterone treatment was efficient in achieving the goal. Yet, complete reliance on day-to-day ovarian ultrasonography to determine the optimum time for induction of ovulation/mating in superovulated camels remained the key limiting factor for application of ART's in this specie. Lately, Khalifa et al. (2016) introduced a reliable fixed-time induction of synchronized multiple ovulation and insemination regimen during the reproductive transition period in Egypt (mid-October to midNovember). This regimen encompassed delivering equine Chorionic Gonadotropin (eCG, $2500 \mathrm{IU}$ i.m) in serial decreasing doses over three consecutive days, following a 13-days period of progesterone priming, and performing induction of ovulation by 5000 IU human chorionic gonadotropin (hCG) twelve days later (i.e. at peak of the mature phase of the ovarian cycle). On the other hand, Monaco et al. (2017) highlighted the benefit of epidural administration of Lecirelin, a synthetic GnRH analogue, to induce ovulation in dromedaries' synchronized ovulation schemes. Most recently, Khalifa et al. (2019) appraised the efficiency of two hormonal regimens on inducing synchronized multiple ovulation in dromedary females at the beginning of the non-breeding season (April - May) in Egypt.

\section{Non-conventionally-induced multiple ovulation}

Generally, induced superovulation by means of hormonal treatments is commonly associated with several restrictions least of which are the amount of time and labor involved, high incidence of retained follicles, as well as substantial individual variations in ovarian response and quality of recovered embryos. The major impediment here is that repeated hormonal superovulation develops refractoriness to the treatment due to formation of antibodies against the exogenous gonadotropin. Last but not least, information is still scarce regarding the time required for a superovulated animal to reinstitute its hormonal balance before a subsequent superovulation treatment could be considered (Holtz, 2005; Anouassi and Tibary, 2013). Therefore, several studies were conducted to establish alternatives to conventional hormonal superovulation.

In this context, either active or passive immunization against endogenous inhibin, a disulphide-linked heterodimeric glycoprotein belonging to the transforming growth factor- $\beta$ (TGF$\beta$ ) superfamily of cytokines and secreted by both granulosa and theca cells (Knight, 1996), has been reported to induce superovulation, increase ovulation rates and embryos collected in different species. Lately, Rateb et al. (2015) investigated the efficiency of neutralizing endogenous inhibin on promoting camel ovarian dynamics during the transition period (mid-August to mid-December) in Egypt. The results illustrated that active immunization against inhibin altered the reproductive hormones patterns, stimulated ovarian follicular activity and assisted the females to override seasonal anestrus prior to the onset of the breeding season. Even further, subsequent long-term observations revealed that active immunization against inhibin developed extended ovarian hyperactivity after 5 months from initiating the treatment (Rateb et al., 2016).The authors also established a hormonal regimen to control the ovarian hyper-stimulation and synchronize ovulation time in the immunized camels.

\section{In vitro embryo production}

Effective development of in vitro embryo production depends mainly on acquiring functionally-competent oocytes followed by adequate preparation through in vitro maturation process. On the other side, in vitro preparation of spermatozoa for fertilization is the other fundamental step that complements in vitro fertilization procedure. In vitro embryo production technology has been successfully applied to different animal species with varying rates of success. In camels, previous results indicated that $14-23 \%$ of in vitro-produced embryos attained the blastocyst stage when fresh semen was used for fertilization. This percentage is commonly reduced 
drastically when either frozen-thawed or epididymel spermatozoa were used. Recently, El-Sayed et al. (2015) evaluated the effect of two sources of spermatozoa; i.e. epididymal and frozen-thawed spermatozoa, accompanied by two fertilization techniques (intra-cytoplasmic sperm injection (ICSI) or conventional in vitro fertilization) on cleavage rates and development of produced dromedary embryos and promising results were obtained.

\section{Futuristic Prospects}

Despite the rapid development and marked achievements that occurred throughout the past decade to improve dromedary reproductive performance, further topics still need to gain focus of upcoming research. For example, optimizing preparation and maturation of oocytes, spermatozoa's capacitation medium as well as culture media is still imperious to maximize quantity and quality of in vitro-produced embryos. Furthermore, developing adequate procedures for selection and separation of fertile spermatozoa from within the whole ejaculate will surely contribute in increasing the outcomes of $\mathrm{AI}$ and IVF in this specie. Even further, establishing a reliable procedure for embryo cryopreservation will broaden in-field application of embryo transfer in camels.

\section{REFERENCES}

Anouassi A. and A. Tibary, 2013. Development of a large commercial camel embryo transfer program: 20 years of scientific research. Anim. Reprod. Sci. (136): 211-221.

Deen A. and E. El-Hassanein, 2013.Effect of gonadotrophin treatment on circulating estradiol and progesterone profiles, growth and maturation of follicles and embryo collection in female camels for two successive superovulation trials. Camel: Int. J. Vet. Sci. (1): 97-113.

El-Bahrawy K.A., 2010a.Effect of seminal plasma centrifugation for viscosity elimination on cryopreservation of dromedary camel semen. Nature and Science (8): 196-202.

El-Bahrawy K.A., 2010b. Cryopreservation of dromedary camel semen supplemented with $\alpha$ amylase enzyme. J. Camel Pract. Res. (17): 1-6.

El-Bahrawy K.A., 2017a.The influence of caffeine supplementation and concerted utilization of enzymatic and mechanical semen liquefaction on freezability of dromedary camel spermatozoa. Int. J. Vet. Sci. Med. (5): 121-127.

El-Bahrawy K.A., 2017b.Influence of enzymatic and mechanical liquefaction of seminal plasma on freezability of dromedary camel semen. World's Vet. J. (7): 108-116.

El-Bahrawy K.A., El-Hassanein E.E. and S.A. Rateb, 2012.Effect of collection frequency, extender and thawing temperatures on the motility recovery of cryopreserved dromedary camel spermatozoa. J. Anim. Poult. Prod. (3): 73-82.
El-Bahrawy K.A., El-Hassanien E.E. and Y.M. Kamel, 2010.Comparison of gentamicin and ciprofloxacin in dormedary camels' semen extender. World J. Agric. Sci. (6): 419-424.

El-Bahrawy K.A., El-Hassanien E.E., Fateh El-Bab A.Z. and M.M. Zeitoun, 2006.Semen characteristics of the male camel and its freezability after dilution in different extenders.Int. Sci. Camel Conf., El-Qaseem, KSA.Pp, 2037-2053.

El-Bahrawy K.A., Khalifa M.A. and S.A. Rateb, 2015. Recent advances in dromedary camel reproduction: an Egyptian field experience. Emir. J. Food Agric. (27): 350-354.

El-Bahrawy K.A., Rateb S.A., Khalifa M.A., Monaco D. and G.M. Lacalandra, 2017.Physical and kinematic properties of cryopreserved camel sperm after elimination of semen viscosity by different techniques.Anim. Reprod. Sci. (187): 100-108.

El-Hassanein E.E., 2003.An invention for easy semen collection from dromedary camels, El-Hassanein Camel Dummy. Recent Advances in Camelid Reproduction, L. Skidmore and G.P. Adams (Eds.) Publisher: Int. Vet. Inf. Service (www.ivis.org), Ithaca, New York, USA.

El-Sayed A., Ashour G., Kamel A.M. and K. A. ElBahrawy, 2015.Assessment of embryo production of dromedary (Camelus dromedarius) using two semen sources and two in vitro fertilization techniques. Egyptian J. Anim. Prod. (52): 153160.

Holtz W., 2005. Recent developments in assisted reproduction in goats. Small Rumin. Res. (60): 95-110.

Kershaw-Young C.M., Stuart C., Evans G.and W.M. Maxwell, 2013. The effect of glycosaminoglycan enzymes and proteases on the viscosity of alpaca seminal plasma and sperm function. Anim. Reprod. Sci. (138): 261-7.

Khalifa M.A., Abd El-Hamid I.S. and S.A. Rateb, 2019.Induction of synchronized multiple ovulation in dromedary camels during the early non-breeding season. Small Rum. Res. (in press).

Khalifa M.A., Rateb S.A. and K.A. El-Bahrawy, 2016. Fixed-time induction of ovulation in camels superovulated by different eCG modalities during the transition period in Egypt. Trop. Anim. Health Reprod. (48): 823-829.

Knight P.G., 1996. Roles of inhibins, activins and follistatin in the female reproductive system.Frontiers of Neuroendocrinology. (17): 476-509.

Monaco D., Lacalandra G.M. and K.A. El-Bahrawy, 2017. Epidural administration of Lecirelin for inducing ovulation in female dromedary camels (Camelus dromedarius): A preliminary study. Small Rum. Res. (146): 69-71.

Mosaferi S., Niasari N.A., Abarghani A., Gharahdaghi A.A. and A. Gerami, 2005. Biophysical and biochemical characteristics of 
bactrian camel semen collected by artificial vagina. Theriogenology (63): 92-101.

Padalino B., Rateb S.A., Ibrahim N.H., Monaco D., Lacalandra G.M. and K.A. El-Bahrawy, 2016. Behavioral indicators to detect ovarian phase in the dromedary she-camel. Theriogenology (85): 1644-1651.

Rateb S.A., 2016. Ultrasound-assisted liquefaction of dromedary camel semen. Small Rum. Res. (141): 48-55.

Rateb S.A., El-Bahrawy K.A. and M.A. Khalifa, 2016. The prolonged reproductive response to immunization against inhibin and manipulating ovarian hyperactivity for timed ovulation in camels. Small Rum. Res. (137): 53-58.

Rateb S.A., El-Hassanein E.E., El-Koumy A.G., ElBahrawy K.A. and Z.R. Abo El-Ezz, 2011. Manipulation of Reproductive Hormones Disorder in Sub-Fertile Male Dromedary Camels
Using Exogenous Gonadotropic-Releasing Hormone (GnRH). World J. Agric. Sci. (7): 280285.

Rateb S.A., Khalifa M.A. and K.A. El-Bahrawy, 2015. The influence of active immunization against inhibin on dromedarycamel ovarian and hormonal dynamics. Small Rum. Res. (132): 3236.

Rateb S.A., Monaco D., El-Bahrawy K.A., Khalifa M.A., Abd El-Hamid I.S., Kamel A.M., Accogli G., Lacalandra G.M. and S. Desantis, 2019. Ramifications of protease-based liquefaction of camel semen on physical, kinematic and surface glycopattern of cryopreserved spermatozoa. Anim. Reprod. Sci. (208): 106-121.

Saad H.M., Rashed M.A., Abdalla E.B. and S.A. Rateb, 2019. Molecular characterization of dromedary camel potential fertility: A new approach. Small Rum. Res. (170): 31-36. 